\title{
Mesenchymal Stem Cells Suppress Muscle Atrophy Induced by Hindlimb Suspension
}

\author{
Mi Jin Kim ${ }^{1 \dagger}$, Yong Min Kim† ${ }^{4 \dagger}$ Z-Hun Kim¹, Si-Hyun Heo ${ }^{1}$, Sun-Mi Kim¹, Jung-Wook Hwang ${ }^{1}$, Woo-Jin Chang ${ }^{2,3}$, Min Jung Baek and Yong- \\ Soo Choi ${ }^{* *}$ \\ ${ }^{1}$ Department of Applied Bioscience, CHA University, Seongnam, Korea \\ ${ }^{2}$ Department of Mechanical Engineering, University of Wisconsin-Milwaukee, Milwaukee, USA \\ ${ }^{3}$ Great Lakes WATER Institute, University of Wisconsin-Milwaukee, Milwaukee, USA \\ ${ }^{4}$ Department of Obstetrics and Gynecology, Bundang CHA Hospital, Seongnam, Korea \\ +Contributed equally to this study
}

\begin{abstract}
Objective: The protective effects of human mesenchymal stem cells (MSCs) isolated from different sources (bone marrow, adipose tissue and umbilical cord) on skeletal muscle regeneration were investigated using a hindlimb suspension (HS)-induced muscle atrophy rodent model.

Methods: Female SD rats were randomly assigned to three groups: control, hindlimb suspension (HS), and hindlimb suspension and reloaded (HR). After induction of muscle atrophy for two weeks, MSCs $\left(1 \times 10^{6}\right.$ cells) were injected into the soleus muscle in the HS and HR groups. Two weeks later, the changes of muscle mass, crosssectional area (CSA), muscle-specific gene expression, lactate accumulation and muscle atrophy signaling pathwayrelated proteins were compared.

Results: In the MSCs-injected groups, there was an increase in soleus muscle mass, CSA and expression of skeletal muscle actin and desmin, as well as a decrease in lactate accumulation. All MSCs play an effective role in improvement of the muscle state from the muscle atrophy condition. Especially, HR groups accompanied by normal activity after MSCs injection showed synergic effect on recovery of atrophic muscle. Furthermore, treatment with the MSCs activated the PI3K/AKT pathway known as major signaling of muscle atrophy, consequently reducing expression of the muscle-specific RING finger protein-1 (MuRF-1) and atrophy F-box (MAFbx/Atrogin-1).
\end{abstract}

Conclusions: The results can serve as fundamental data toward the development of new medical strategies for the rapid rehabilitation and regeneration of atrophied muscles.

Keywords: Hindlimb suspension; Mesenchymal stem cell; Muscle atrophy; Muscle regeneration; Skeletal muscle; Soleus muscle; Umbilical cord

\section{Introduction}

Muscle atrophy and weakness can be a consequence of both reduced muscle use (e.g., muscle unloading, exposure to microgravity, immobilization and denervation) and a variety of disease states (e.g., cancer, diabetes and cachexia) [1]. When muscle atrophy occurs, slowtwitch muscle fibers start to decrease in size compared with fast-twitch muscle fibers. As one of the anti-gravity muscles, the soleus muscle is largely composed of slow-twitch fibers, and the contraction of this muscle is known to be critically important for maintaining posture and balance [2].

To date, electronic stimulation, exercise and chemical treatments have been used for the treatment and prevention of muscle atrophy. However, while the resulting muscle regeneration is limited, these treatments also may not be fundamental cures supporting muscle regeneration [2]. In particular, it may be difficult or impossible for patients who are overweight, undergo prolonged bed rest, or display seriously progressed muscle atrophy to perform such treatments. Recent studies have demonstrated that mesenchymal stem cells (MSCs) therapy have the potential to enhance muscular regeneration [3,4]. MSCs are known to secrete angiogenic factors, mitogenic factors and antiapoptotic factors, inducing myogenesis and angiogenesis in a similar fashion as insulin-like growth factor-1 (IGF-1), vascular endothelial growth factor (VEGF), adrenomedullin (AM), and hepatocyte growth factor (HGF) [5,6]. In addition, MSCs have proven to be an effective therapeutic agent for musculoskeletal tissues in experimental models
[7-9]. With such potential displayed by MSCs, they have been applied to the treatment of muscular diseases.

Muscle changes have been observed in a hindlimb suspension (HS) induced muscle atrophy rodent model [10]. HS-induced animal models are frequently used to study the cellular and molecular mechanisms underlying skeletal muscle atrophy by suspending the hindquarters of the animal and preventing the hindlimb from participating in normal weight bearing $[10,11]$. In this study, we tried to compare and analyze the efficacy of MSCs derived from 3 different human sources to target for the muscle atrophy using a HS-induced animal model. In addition, we examined the effect of MSCs on muscle regeneration and muscle atrophy signaling pathway. From the results, we found that MSCs could suppress loss of muscle as well as accelerate muscle regeneration.

*Corresponding author: Yong-Soo Choi, Ph.D, Department of Applied Bioscience, CHA University, (CHA Bio Complex) 689, Sampyeong-dong, Bundanggu, Seongnam-si, Gyeonggi-do 463-400, Korea, Tel: 82-31-881-7232; Fax: 82-31881-7231; E-mail: yschoi@cha.ac.kr

Min Jung Baek, M.D, Department of Obstetrics and Gynecology, Bundang CHA Hospital, 59, Yatap-ro, Bundang-gu, Seongnam-si, Gyeonggi-do, 463-712, Korea, Tel: 82-31-780-5298; E-mail: goodgood75@naver.com

Received January 19, 2015; Accepted February 12, 2015; Published February 14,2015

Citation: Kim MJ, Kim YM, Kim ZH, Heo SH, Kim SM, et al. (2015) Mesenchymal Stem Cells Suppress Muscle Atrophy Induced by Hindlimb Suspension. J Stem Cell Res Ther 5: 266. doi:10.4172/2157-7633.1000266

Copyright: ( $2015 \mathrm{Kim} \mathrm{MJ}$, et al. This is an open-access article distributed under the terms of the Creative Commons Attribution License, which permits unrestricted use, distribution, and reproduction in any medium, provided the original author and source are credited. 
These results imply that MSCs can be used as promising agents for the treatment of patients suffering from muscle atrophy.

\section{Materials and Methods}

\section{Cell preparation}

Human adipose-derived mesenchymal stem cells (AD-MSCs; PCS500-011, passage \#6) were purchased from the American Type Culture Collection (ATCC, Manassas, VA, USA) and cultured in Dulbecco's modified Eagle medium (DMEM; Hyclone laboratories, Inc., Logan, UT, USA) supplemented with $10 \% \mathrm{FBS}, 100 \mathrm{mg} / \mathrm{mL}$ streptomycin, and $100 \mathrm{IU} / \mathrm{mL}$ penicillin. Human bone marrow-derived mesenchymal stem cells (BM-MSCs; MSC-001F, passage \#7) were purchased from Stem Cell Inc. (Vancouver, Canada) and maintained in the same culture conditions as the AD-MSCs, described above. The isolation and culture of human umbilical cord-derived mesenchymal stem cells (UC-MSCs) were performed as described in our previous study [12]. UC-MSCs at passage 4-5 were used in all experiments.

\section{Myogenic differentiation of MSCs with 5 '-azacytidine}

To confirm whether chemical induction could trigger the differentiation of MSCs derived from multiple sources (UC-MSCs, $\mathrm{AD}-\mathrm{MSCs}$ and BM-MSCs) into muscle cells, the MSCs were exposed to different time courses $\left(12,24,36\right.$ and $72 \mathrm{~h}$ ) of $10 \mu \mathrm{M} 5^{\prime}$-azacytidine (Sigma-Aldrich, St. Louis, MO, USA) on day 3 of culture $[13,14]$. The cells were renewed each day with fresh media containing 5 '-azacytidine. To test the 5 '-azacytidine-induced differentiation of the MSCs, the expression of MyoD and Pax-7, markers of muscle differentiation, were evaluated via analysis, as described below.

\section{Experimental animals and hindlimb-suspension (HS)}

All procedures for animal care and treatment were performed in accordance with the Care and Use of Laboratory Animals Guidelines from CHA University (Seongnam, Korea), and all protocols were approved by the Animal Experimentation of CHA University Committee (Approval No.: 130012).

Female Sprague-Dawley (SD) rats at 5 weeks of age (Koatech Co., Pyeongtaek, Korea) were used in this study. The animals were randomly assigned to the control, hindlimb suspension (HS), and hindlimb suspension and reloaded group (HR). HS group represents people who are lied down on bed, continuously in situations with no or less activity (for example, patient that are in long bed rest). In the case of HR group which reflecting patient who return to the normal life after experiencing muscle atrophy. Hindlimb unloading was induced as described in the previous report [15]. Rats in the HS and HR groups were each randomly divided into four subgroups. After 2 weeks of HS, the HS groups were divided as follows: (1) saline injection (HS+Saline); (2) UC-MSCs injection (HS+UC); (3) AD-MSCs injection (HS+AD); and (4) BMMSCs injection (HS+BM). Similarly, the HR groups were divided after 2 weeks of HS as follows: (1) reloaded + saline injection (HR+Saline); (2) reloaded+UC-MSCs injection (HR+UC); (3) reloaded + AD-MSCs injection $(\mathrm{HR}+\mathrm{AD})$; and $(4)$ reloaded $+\mathrm{BM}-\mathrm{MSC}$ injection $(\mathrm{HR}+\mathrm{BM})$. MSCs were injected directly into the soleus muscle, and the animals were sacrificed at 1 and 2 weeks post-injection for observation of the effects (Figure 1).

To simulate suspension conditions in the rat muscles, a hindlimb suspension apparatus that lifts the back legs off the ground was utilized. Dressing tape was used to stabilize the tail at the top of the cage so that the legs could be maintained in a suspended condition. Front leg movement was allowed to minimize the stress levels in the rats.
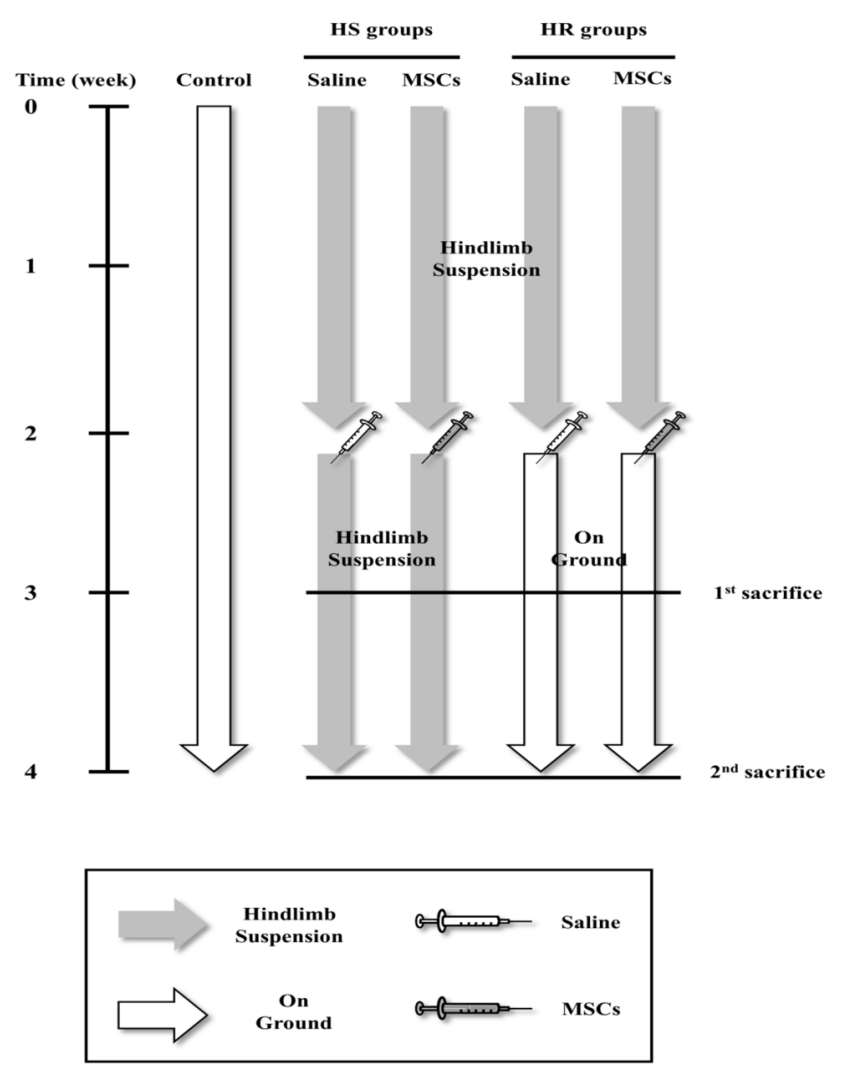

Figure 1: Summary of the experimental design to determine the effects of MSCs on hindlimb suspension (HS)-induced muscle atrophy in SpragueDawley rats.

Furthermore, the body angles of the rats were set at 30 degrees with respect to the floor (Supplemental Figure 1).

\section{Transplantation of MSCs into soleus muscles}

To evaluate the efficacy of the three types of MSCs, each type was injected after 2 weeks of HS. All experimental groups $\left(1 \times 10^{6} \mathrm{cells} / \mathrm{limb}\right.$ in $50 \mu \mathrm{L}$ saline) underwent injection directly into both soleus muscles, and the efficacy was observed 1 and 2 weeks later. Saline groups were injected with an equivalent volume of $\mathrm{NaCl}$ solution $(0.9 \% \mathrm{w} / \mathrm{w})$.

\section{Histological and cross-sectional area (CSA) analysis of soleus muscles}

Tissue samples for microscopy and immunoperoxidase staining were fixed in $4 \%$ paraformaldehyde solution (Biosesang Inc., Seongnam, Korea). The samples were then dehydrated and embedded in paraffin. The histological cross-sections $(5 \mu \mathrm{m})$ were stained with hematoxylin and eosin (H\&E) (Sigma-Aldrich), and then examined under a light microscope (Nikon ECLIPSE E600, Fukuoka, Japan) to calculate the cross-sectional area (CSA) of muscle fibers using the previously reported method [16]. We obtained 10 sections of muscle from each sample group and randomly selected 100 fibers for each section, then measured CSA $\left(\mu \mathrm{m}^{2}\right)$ using the Image J Software (by Broken Symmetry Software). The measured CSA was converted into fold change (\%).

\section{Protein extraction and lactate concentration analysis}

The amount of lactate in the soleus muscle was measured using a Lactate colorimetric assay kit (BioVision Inc., Mountain view, CA, 
USA). Proteins were extracted from the soleus muscles by placement in RIPA buffer (Rockland Inc., Pottstown, PA, USA) supplemented with a protease inhibitor cocktail (Quartett Immunodiagnostika, Berlin, Germany). The samples were disrupted with a PT 1200E homogenizer (Kinematica, Luzern, Switzerland), and the protein concentration was determined via the Bradford protein assay (Bio-Rad Laboratories Inc., Hercules, CA, USA) using bovine serum albumin (BSA; Thermo Scientific, Waltham, MA, USA) as a standard. For the lactate assay, $990 \mu \mathrm{L}$ of lactate assay buffer and $10 \mu \mathrm{L}$ of sample were added to each well of a 96-well plate (Nunc, Thermo Scientific, Roskilde, Denmark). Next, $50 \mu \mathrm{L}$ of the reaction mix was slowly added to the mixture, and the reaction was allowed to proceed for $30 \mathrm{~min}$ at room temperature. The absorbance was then measured at $570 \mathrm{~nm}$, and the sample concentrations were calculated within the range of the standard curve using a microplate reader (Epoch, Bio-Tech. Inc., Winoski, CA, USA).

\section{Quantitative real-time PCR analysis}

For each sample of soleus muscle, total RNA was isolated using the easy-spin ${ }^{\mathrm{TM}}$ total RNA extraction kit (iNtRON Biotechnology Inc., Seoul, Korea), according to the manufacturer's instructions, and $1 \mu \mathrm{g}$ of the total RNA was then reverse transcribed using Reverse Transcription 5X Master Premix (ELPIS Biotech, Daejeon, Korea), followed by PCR amplification. Transcript levels were determined by quantitative realtime PCR (qPCR) on the ABI PRISM 7000 sequence detection system (Applied Biosystems, Foster City, CA, USA), using SYBR Green I as a double-stranded DNA-specific dye, according to the manufacturer's instructions. PCR was carried out using $5 \mu \mathrm{M}$ of cDNA, $10 \mu \mathrm{M}$ of SYBR Green PCR master mix, and $5 \mathrm{pM}$ of sense and anti-sense primers for skeletal muscle actin (sense: 5'-TTG AGA CCT TCA ACG TGC C-3', anti-sense: 5'-CCA GAA TCC AAC ACG ATG C-3') and desmin (sense: 5'-CTG ATA GAC GAC CTG CAG A-3', anti-sense: 5'-CAG AGT GGC TGC ATC CAC-3') in a final volume of $20 \mu \mathrm{L}$ per reaction. Primer concentrations were determined via preliminary experiments to analyze the optimal concentrations required. At least three independent PCR tests were performed to allow statistical analyses. The amounts of PCR products detected were normalized to the house-keeping gene, GAPDH (sense: 5'-TGA AGG TCG GTG TGA ACG-3', anti-sense: 5'-TGA TGG CAA CAA TGT CCA C-3'), to determine the relative expression ratios of each mRNA in relation to the control group.

\section{Western blotting}

The soleus muscle was lysed with RIPA buffer containing a protease inhibitor cocktail (Roche Diagnostics, Mannheim, Germany). The extracted proteins were then denatured in sodium dodecyl sulfatepolyacrylamide gel electrophoresis (SDS-PAGE) sample buffer $(62.5$ $\mathrm{mM}$ tris- $\mathrm{HCl}$ [pH 6.8], 2\% SDS, 10\% glycerol, 5\% 2-mercaptoethanol) and boiled for $5 \mathrm{~min}$. After separation of the protein samples $(30 \mu \mathrm{g})$ by $10 \%$ SDS-PAGE, the proteins were transferred onto nitrocellulose membranes (Whatman, Dassel, Germany) for $3 \mathrm{~h}$ at $4^{\circ} \mathrm{C}$. The membranes were then washed with phosphate buffered saline (PBS; Biosesang Inc.) supplemented with $0.1 \%$ Tween-20 (Biosesang Inc.) and blocked with blocking solution (3\% BSA and with $0.1 \%$ Tween-20 in PBS) at room temperature. The membranes were incubated overnight with each primary antibody at $4^{\circ} \mathrm{C}$. Detection was performed using antiphospho-Akt, 1:1000 (Cell signaling technology, Beverly, MA, USA; \#9271), anti-phospho-FoxO1/FoxO3a, 1:1000 (Cell signaling; \#9464), anti-FoxO1, 1:1000 (Cell signaling; \#2880), anti-FoxO3a, 1:1000 (Cell signaling; \#2497), anti-Akt, 1:1000 (Santa Cruz Biotechnology, Inc., Santa Cruz, CA, USA; sc-8312), anti-MuRF-1, 1:1000 (Santa Cruz; sc-27642), anti-MAFbx, 1:1000 (Santa Cruz; sc-27645), anti-MyoD, 1:1000 (Santa Cruz; sc-31942), anti-Pax-7, 1:1000 (Santa Cruz; sc-7749) and anti- $\beta$-actin, 1:2000 (Santa Cruz; sc-47778). After washing the blots with PBS-T, incubation was carried out with horseradish peroxidase (HRP)-conjugated secondary goat anti-rabbit, 1:5000 (Santa Cruz; sc-2004), goat anti-mouse, 1:5000 (Santa Cruz; sc-2005) and donkey anti-goat antibodies, 1:5000 (Santa Cruz; sc-2020), corresponding to each primary antibody. Finally, the target proteins were visualized by enhanced chemiluminescence detection (ECL component from Pierce Clarity ${ }^{\mathrm{TM}}$ and Western ECL Substrate from Bio-Rad).

\section{Double-immunofluorescence analysis for determination of muscle regeneration}

Immunofluorescence analysis was performed on paraffin sections of soleus muscles from the control, HS+Saline, HS+UC and HR+UC group rats at 2 weeks using primary anti-Desmin, 1:100 (Abcam plc, Cambridge, UK; ab15200), and anti-human nuclei antibodies, 1:100 (Millipore, Billerica, MA, USA; MAB1281). The sections were rehydrated and washed in $0.1 \mathrm{M}$ PBS before incubation with $10 \%$ normal serum (Vector Laboratories Inc., Burlingame, CA, USA) at room temperature to block nonspecific staining. The tissue sections were then incubated overnight at $4^{\circ} \mathrm{C}$ in PBS containing primary antibody and 5\% normal serum. For visualization the primary antibodies, the sections were incubated with Cy3-conjugated goat anti-rabbit IgG, 1:300 (Invitrogen, Carlsbad, CA, USA), and Alexa Fluor 488-conjugated goat anti-mouse IgG, 1:300 (Invitrogen), for $1 \mathrm{~h}$ at room temperature in the dark. The nucleus was counterstained with DAPI (Vector laboratories), and the cells were visualized via confocal microscopy (TCS SP5 II, Leica, Heidelberg, Germany) with $20 \times 10$ NA objectives. Digital photographs were obtained using Leica LAS AF Ver 2.6 software (Fuji photo film Co., Tokyo, Japan).

\section{Statistical analysis}

All in vitro experiments were independently performed at least three times. Data were analyzed using the SigmaPlot 11.0 software (Systat Software, San Jose, CA, USA). When comparing more than two groups, one-way analysis of variance (ANOVA) combined with the Bonferroni post hoc test was used. For comparison of two samples, Student's $t$-test was used. Quantitative results are shown as the mean \pm standard deviation for each experiment. $\mathrm{P}<0.05$ was considered statistically significant.

\section{Results}

\section{Expression of muscle-specific markers is higher in UC-MSCs after myogenic differentiation}

After exposure of the three types of MSCs (UC-MSCs, AD-MSCs and BM-MSCs) to $5^{\prime}$-azacytidine, the expression of muscle-specific markers (MyoD and Pax-7) were analyzed over time (Figure 2A). The expression of the markers increased continuously in the UC-MSCs, by over 2 -fold in $72 \mathrm{~h}$ (Figures $2 \mathrm{~B}$ and $2 \mathrm{C}$ ). In contrast, significant change of the markers was not observed in the $\mathrm{AD}$ - and BM-MSCs.

\section{MSCs suppress the loss of muscle mass and decrease lactate concentration}

The soleus muscles were extracted from the rats at 1 and 2 weeks post-injection with MSCs for measurement of the muscle mass. The muscle mass was normalized with respect to the body weight of the corresponding rat (Figure 3A; $\mathrm{p}<0.05$ ). Compared with the control group, the HS+Saline group demonstrated a significantly decrease $(51.2 \%)$ in muscle mass. In contrast, the HS+UC group had a significantly $33 \%$ increase of muscle mass compared to the HS+saline group after 
Citation: Kim MJ, Kim YM, Kim ZH, Heo SH, Kim SM, et al. (2015) Mesenchymal Stem Cells Suppress Muscle Atrophy Induced by Hindlimb Suspension. J Stem Cell Res Ther 5: 266. doi:10.4172/2157-7633.1000266

A

\begin{tabular}{|c|c|c|c|c|c|c|c|c|c|c|c|c|c|c|}
\hline \multirow[b]{2}{*}{$5^{\prime}$-azacytidine (h): } & \multicolumn{4}{|c|}{ UC-MSCs } & \multicolumn{5}{|c|}{ AD-MSCs } & \multicolumn{5}{|c|}{ BM-MSCs } \\
\hline & 12 & 24 & 36 & 72 & $\mathbf{0}$ & 12 & 24 & 36 & 72 & $\mathbf{0}$ & 12 & 24 & 36 & 72 \\
\hline MyoD & $-\infty$ & 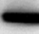 & - & 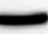 & - & & - & - & $=$ & - & - & $=$ & - & - \\
\hline Pax-7 & $-\cdots$ & - & - & - & - & & - & & - & - & & & & \\
\hline$\beta$-actin & & & & & & & & & & & & & & \\
\hline
\end{tabular}

B

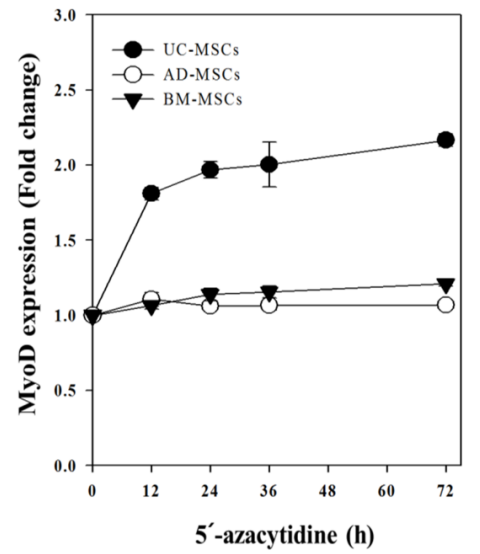

C

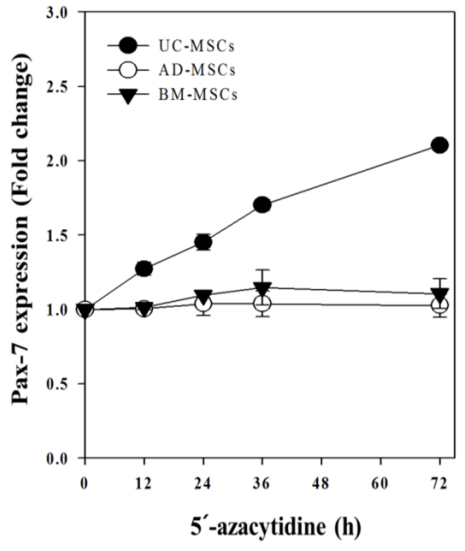

Figure 2: Comparative analysis of the myogenic differentiation capability after the treatment of three types of MSCs with $5^{\prime}$-azacytidine for $72 \mathrm{~h}$. (A) Expression of MyoD and Pax-7 were quantified by performing densitometry on Western blots and normalizing to $\beta$-actin protein levels. A positive linear correlation was observed between the $5^{\prime}$-azacytidine treatment time and changes in protein expression levels. (B and $\mathrm{C}$ ) Error bars represent the standard error of the mean.

A

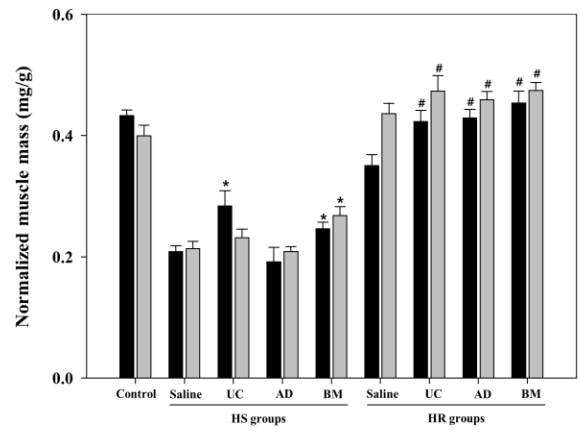

B

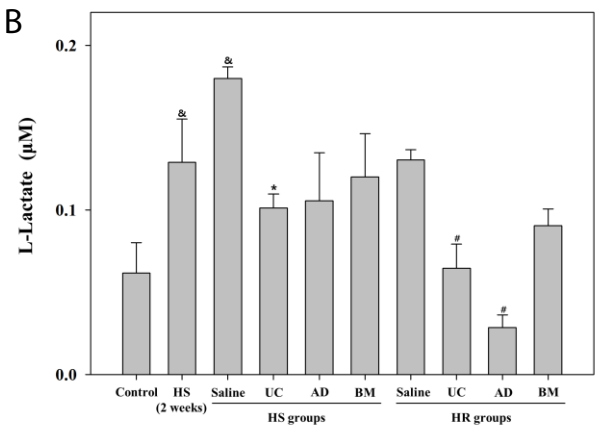

Figure 3: The effect of MSCs on muscle mass and lactate levels in rats with HS-induced muscle atrophy. (A) Soleus muscle weight/body weight (mg/g) at one and two weeks ("P<0.05, compared to HS+Saline group; ${ }^{*} \mathrm{P}<0.05$, compared to HR+Saline group). (B) Lactate levels in the soleus muscle at 1 week after MSCs injection ( ${ }^{\circledR} P<0.05$, compared to control group; ${ }^{*} P<0.05$, compared to the HS+Saline group; ${ }^{\#} P<0.05$, compared to HR+Saline group).

1 week, and the HS+BM group increased $13 \%$ and $26 \%$ after 1 and 2 weeks, respectively. Also, all MSCs injected HR groups had a significant increase in muscle mass compared to the HR+saline group after 1 week. Based on these results, we confirmed that MSCs injection was effective on the recovery of muscle mass in both cases (HS and HR models).

Ischemia of the hindlimb can be observed during HS. To examined this, the lactate concentration in the soleus muscle was analyzed (Figure $3 \mathrm{~B} ; \mathrm{p}<0.05)$. In the soleus muscles at two weeks after HS, the lactate concentration was increased by 2 -fold compared with the control group $(0.12 \pm 0.04 \mu \mathrm{mol} / \mu \mathrm{L}$ vs. $0.06 \pm 0.03 \mu \mathrm{mol} / \mu \mathrm{L})$. After 1 week, the lactate concentration in the HS+Saline group was significantly increased by 3 -fold $(0.18 \pm 0.01 \mu \mathrm{mol} / \mu \mathrm{L})$ than that of the control group. While the lactate concentration in HS+UC group was significantly reduced by $45 \%$ to the control. That in $\mathrm{HR}+\mathrm{UC}$ and $\mathrm{HR}+\mathrm{AD}$ groups were also showed significant reductions ( $50 \%$ and $77 \%$, respectively). Based on these results, we observed that MSCs play an effective role in alleviating accumulation of lactate within the muscle. In all MSCs injected HR groups, the lactate concentrations were exhibited similar or below compared to the level of control.

\section{Atrophic muscles demonstrate faster recovery to normal state in MSCs-injected groups}

To better characterize the muscle mass in hindlimb suspension, histological analysis was conducted and the muscle fiber area of the affected soleus muscles was compared, as shown in Figure 4.

As for the H\&E staining results, the HS+Saline group shown a 
Citation: Kim MJ, Kim YM, Kim ZH, Heo SH, Kim SM, et al. (2015) Mesenchymal Stem Cells Suppress Muscle Atrophy Induced by Hindlimb Suspension. J Stem Cell Res Ther 5: 266. doi:10.4172/2157-7633.1000266

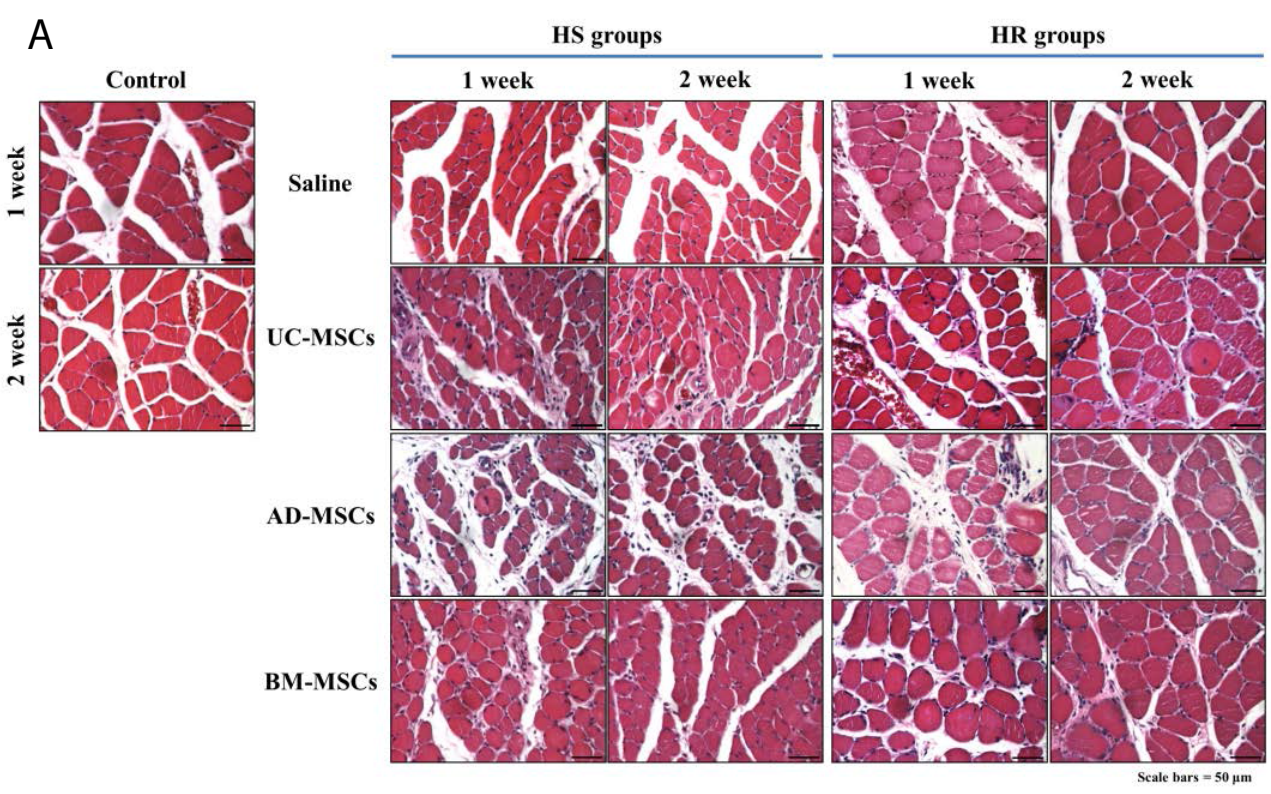

B

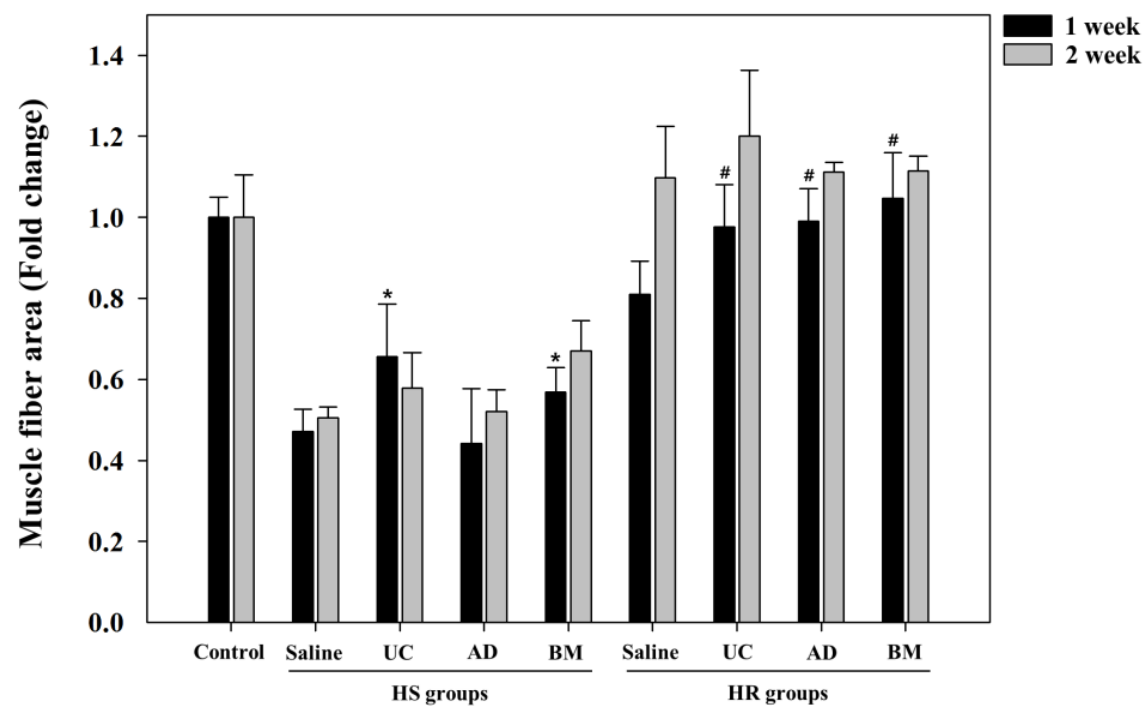

Figure 4: Histological evaluation of MSCs engrafted in soleus muscle. (A) MSCs injection ameliorates the histological phenotype. Muscle sections were stained with hematoxylin and eosin (H\&E). (B) The cross-sectional area (CSA) of soleus muscle fibers. Differences in muscle fiber size were visually confirmed by H\&E staining of the CSA at 1 and 2 weeks ( ${ }^{*} P<0.05$, compared to HS+Saline group; ${ }^{~} \mathrm{P}<0.05$, compared to HR+Saline group). (Original magnification $200 \mathrm{X}$, scale bars=50 $\mu \mathrm{m}$ ).

reduction in muscle fiber size compare to the control group (Figure 4A). Although the size of muscle fibers increased, the number of individual muscle unit has increase with regards to the taken crosssectional sample image. Also, in the HR groups, healing of the muscle can be clearly seen in both 1 and 2 week groups. Especially, we observed that the muscle status in the HR group showed better state with healing of muscle tissue after 2 weeks than that in the control. The size of muscle fibers grew in both the Saline and all MSCs groups.

In addition, we quantified the changes of CSA of muscle fibers in each group and compared each other as shown in Figure 4B. CSA of muscle fibers in the HS+saline group was reduced by $\geq 2$-fold, to the control group. In contrast, the CSA of HS+UC and HS+BM groups were showed slight increments compared to the HS+Saline group (10\% and 5\%, respectively). On the other hand, all MSCs injected HR groups showed remarkable recoveries of the CSA over the HR+Saline group at 1 week after MSCs injection. Also, similar change in CSA was observed in the HR+Saline group as the control group after 2 weeks which are assumed to be a recovery due to activity.

MSCs enhance muscle-specific markers in HS-induced atrophic muscle 
Citation: Kim MJ, Kim YM, Kim ZH, Heo SH, Kim SM, et al. (2015) Mesenchymal Stem Cells Suppress Muscle Atrophy Induced by Hindlimb Suspension. J Stem Cell Res Ther 5: 266. doi:10.4172/2157-7633.1000266

Page 6 of 9

We confirmed a reduction in mRNA level of muscle-specific markers (skeletal muscle actin and desmin) caused by muscle atrophy (Figure $5 \mathrm{~A}$ and $5 \mathrm{~B} ; \mathrm{p}<0.05$ ). Both genes significantly increase in the HS groups by all MSCs (shown similar results in both 1 and 2 weeks). Also, in the HR groups, the expression of desmin significantly increased by $\mathrm{AD}-\mathrm{MSCs}$ at 1 week (Figure $5 \mathrm{~A}$ ), the expression of skeletal muscle actin significantly increased by BM-MSCs at 2 weeks (Figure 5B). Especially, as for the result to be focused on, the expression of both genes showed comparable to the control level by UC-MSCs injection at 2 weeks (Figure 5A and 5B).

\section{MSCs could activate PI3K/AKT signaling in HS-induced atrophic muscle}

We selected UC-MSCs as a representative among all MSCs for further analysis MSCs, and immunofluorescence (Figure 6A) and Western blotting (Figures 6B-6G) were carried out to find out how they affected to muscle atrophy. The change in expression of desmin is shown in Figure 6A. Also, surviving UC-MSCs engrafted soleus muscle via human nuclei marker was observed. Desmin expression in the HS+saline group reduced compared to the control group and showed an additional structural change (collapsing phenomenon). Especially an increase of desmin expression and structural recovery was observed in UC-MSCs injected groups (HS+UC and $\mathrm{HR}+\mathrm{UC}$ ).

Compared with the control group, the HS+Saline group decreased the expression of $\mathrm{p}$-AKT and $\mathrm{p}$-FoxO1/3a, whereas the expression of MAFbx and MuRF-1 were increased (Figures 6B-6G, $p<0.05$ ). Conversely, in the UC-MSC-injected groups, AKT phosphorylation was significantly recovered to $80 \%$ compared to the HS+Saline group (Figure 6B and 6C). Also, the phosphorylation of FoxO1/3a were restored (Figure 6B, 6D and 6E), while the expression of MAFbx and MuRF-1 were decreased ( $18.3 \%$ and $46.5 \%$, respectively) compared to the HS+Saline group (Figure 6B, 6F and 6G). Similar results were also
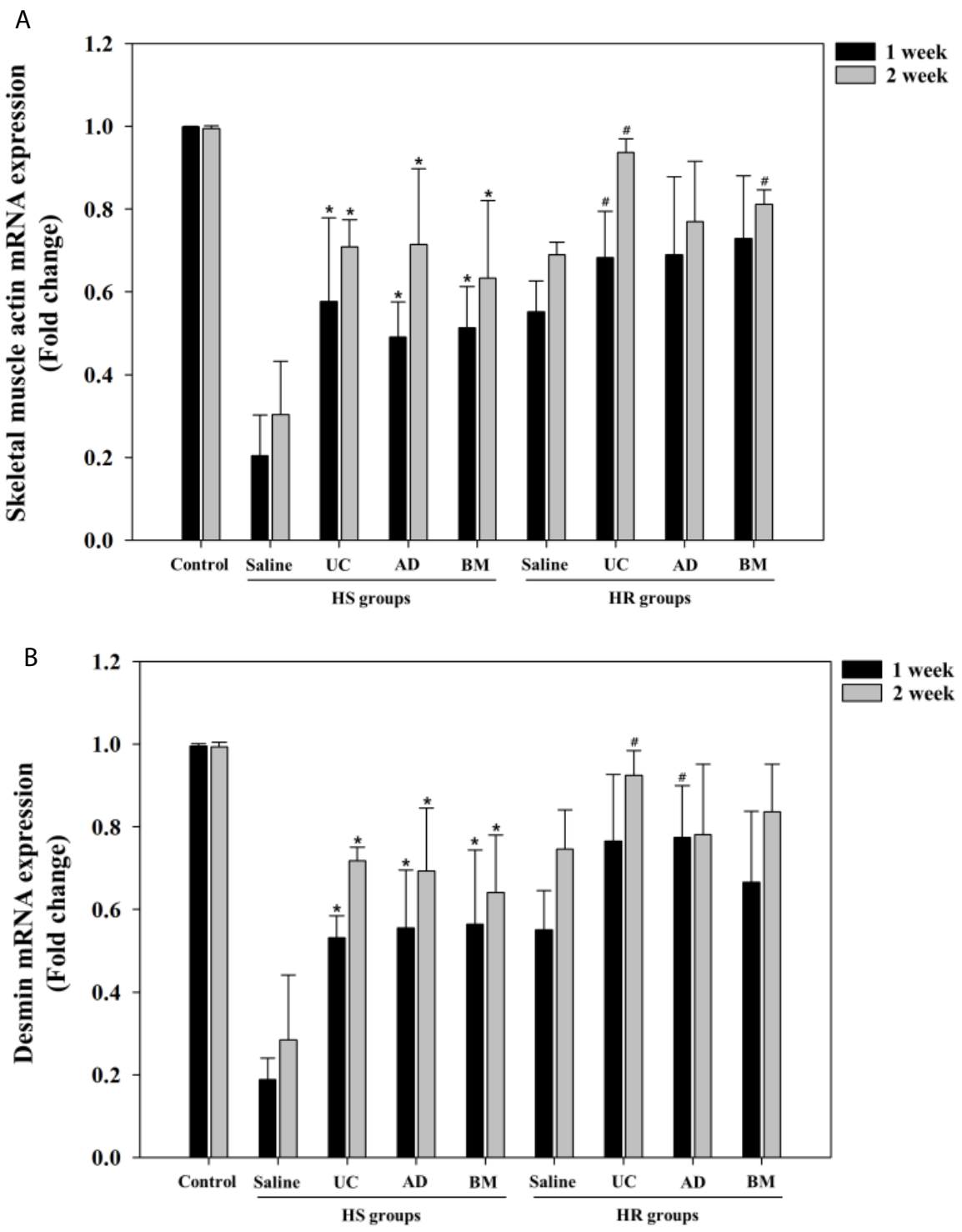

Figure 5: The effect of MSCs on expression of muscle-specific markers in HS-induced atrophic muscle. The mRNA expression of skeletal muscle actin (A) and desmin (B) were confirmed in soleus muscles ( ${ }^{*} \mathrm{P}<0.05$, compared to HS+Saline group; ${ }^{*} \mathrm{P}<0.05$, compared to HR+Saline group). 
Citation: Kim MJ, Kim YM, Kim ZH, Heo SH, Kim SM, et al. (2015) Mesenchymal Stem Cells Suppress Muscle Atrophy Induced by Hindlimb Suspension. J Stem Cell Res Ther 5: 266. doi:10.4172/2157-7633.1000266

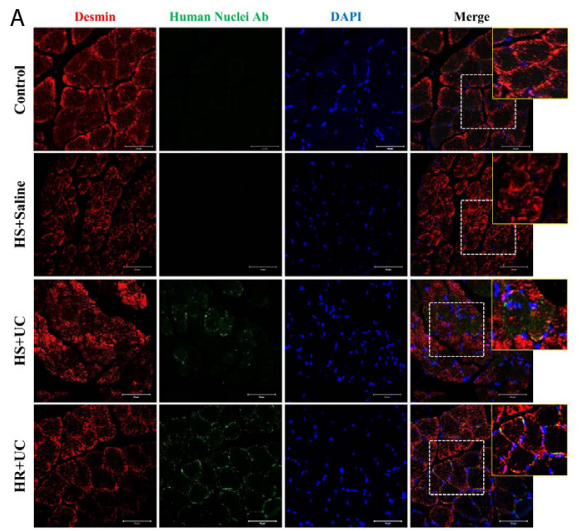

B
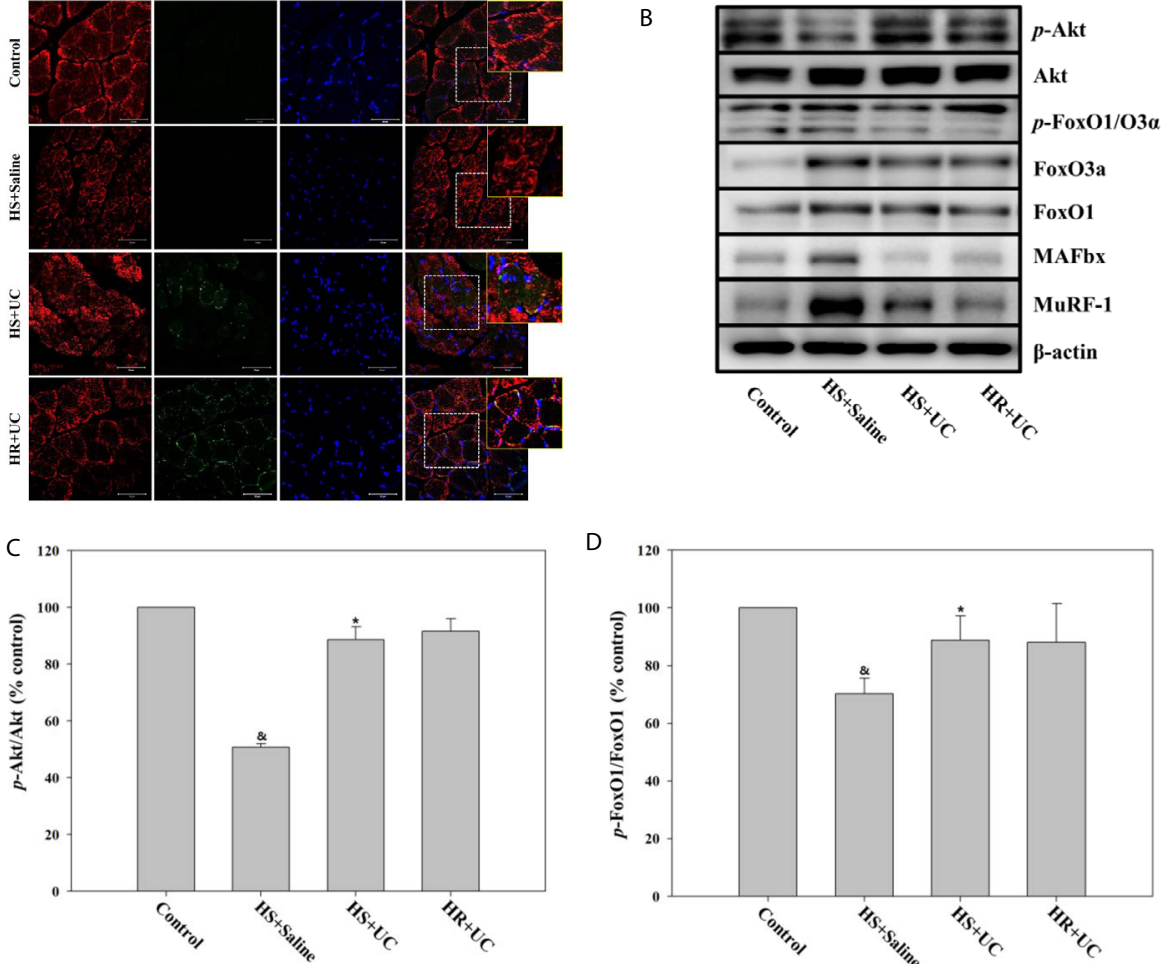

$\mathrm{E}$

$\mathrm{F}$
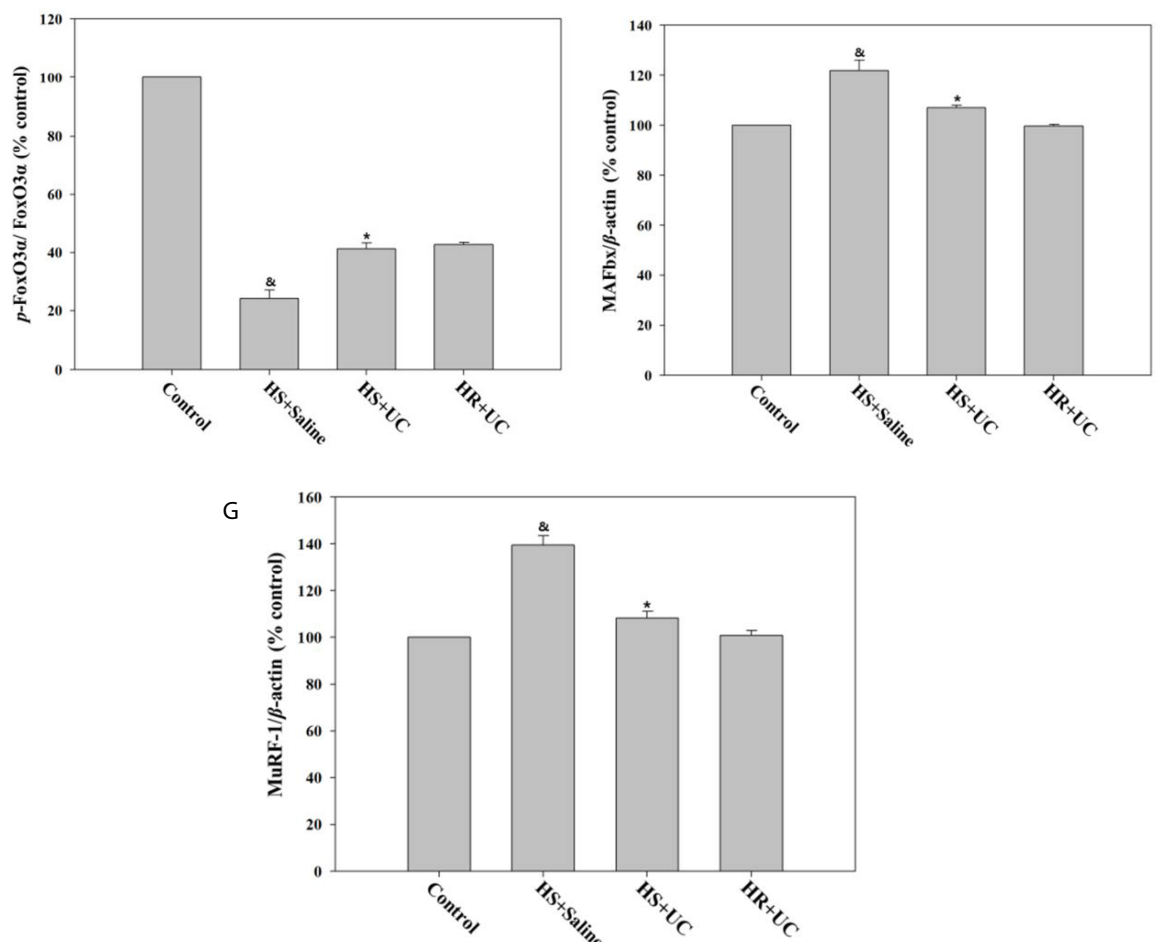

Figure 6: Improvement of muscle regeneration in muscle atrophy after MSCs injection and mechanism analysis.

(A) Immunofluorescence analysis using paraffin sections of soleus muscles from the Control, HS+Saline, HS+UC and HR+UC group rats at 2 weeks. Desmin is labeled with Cy3 (red), UC-MSCs are labeled with alexa 488 (green), and nuclei are stained with DAPI (blue). (Original magnification $200 X$, scale bars $=50$ um). (B-G) Immunoblot analysis of the expression of PI3K/AKT signaling pathway. Representative Western blots showing the expression of whole protein level and phosphorylated $(p)$ forms of AKT and FoxO-1/3a, and the expression of total form of MAFbx (Atrogin-1) and MuRF-1 in the soleus muscle. The expression of phosphorylated AKT (C), FoxO-1/3a (D and E), MAFbx (F) and MuRF-1 (G) normalized to $\beta$-actin expression ( ${ }^{\circledR} \mathrm{P}<0.05$, compared to control group; ${ }^{*} \mathrm{P}<0.05$, compared to HS+Saline group). Error bars represent standard deviation. 
found in the HR groups. Thus, the above findings implied that MSCs are capable of stimulating recovery from muscle atrophy through the regulation of muscle atrophy-related signaling pathway.

\section{Discussion}

Maintenance of muscle mass, which is determined by a balance between protein synthesis and degradation, is essential for the health and survival of humans [2]. Excessive protein degradation in skeletal muscles could induce disuse atrophy of the postural muscles, thereby resulting in decreased muscle mass and strength. The loss of muscle mass has an important regulatory function in determining the outcomes of immobility and weakness [17]. Therefore, studying the interplay of muscle loss and regeneration could increase our understanding of the disease, and possibly lead to the development of promising therapeutics.

Most therapy researches using MSCs were generally carried out using only one or two types of the cells. Even with the same disease, MSCs show different efficacy depending on its origin [7-9,14,18]. Thus, this study tried to compare and analyze the efficacy of MSCs derived from 3 different human organs to target for the treatment of muscle atrophy. Furthermore, we conducted in vivo experiments using not only HS model, reflecting patients who are experiencing muscle atrophy, but also HR model, reflecting patients who return to the normal life after experiencing muscle atrophy, to access the effectiveness and timing of MSCs therapy.

Muscle atrophy is characterized by reduction in muscle mass, muscle strength, protein content and cross section area (CSA), as well as alteration in the type of muscle fiber. Krawiec et al. [19] observed a reduction in the muscle mass as well as the mRNA transcription levels of muscle-specific genes in the postural muscles by disuse conditions. In addition, the atrophy process was induced by a diversity of alterations. Mujika et al. [20] reported that these alterations led to a reduction in the CSA of muscle fiber when the muscle was exposed to atrophic conditions. Consequently, reduction in the CSA could negatively affect muscle strength and motility. Previous studies also showed that the weights of muscle and diameters of myofiber remained significantly smaller in the soleus muscles after HS, and that reduction in myonuclear accretion early in muscle development results in longterm reduction in the muscle size of rat soleus [21]. In this study, we also observed declines of muscle mass (Figure 3A) and CSA (Figure 4B) caused by HS-induced muscle atrophy. Besides, we found the effects of recovery on muscle mass and CSA with time after all MSCs injection. Based on these results, all MSCs showed improving muscle state from muscle atrophic condition.

Lactate produced when ATP is generated via anaerobic metabolism, which occurs due to hypoxia caused by insufficient blood supply to the muscle cells. The lactate accumulation is known to induce changes in muscle contraction proteins that affect muscular strength $[22,23]$. Ischemic conditions were observed in the hindlimb during HS, since the blood circulation might be weakened and restricted. Thus, to elucidate the relationship between muscle lactate accumulation and ischemia, the lactate accumulation in the soleus muscle was measured.

Similarly, we observed that the lactate concentration in soleus muscle was increased by HS-induced muscle atrophy, whereas, $\mathrm{HS}+\mathrm{UC}$ and $\mathrm{HS}+\mathrm{AD}$ groups were significantly exhibited lower lactate accumulation compared with the HS+Saline group after 1 week (Figure 3B). Also, $\mathrm{HR}+\mathrm{UC}$ and $\mathrm{HR}+\mathrm{AD}$ groups were showed significant reductions compare to $\mathrm{HR}+$ Saline group. These results indicate that the injection of MSCs affects lactate level in muscles by suppressing its accumulation.
In addition, we confirmed a reduction in mRNA levels of musclespecific genes (skeletal muscle actin and desmin) caused by muscle atrophy (Figure 5A and 5B). However, both genes significantly increase in MSCs-injected groups compared to Saline groups. Especially, the condition accompanied by normal activity after MSCs injection showed synergic effects on recovery of atrophic muscle.

So, we carefully compared and analyzed the efficacy of all MSCs from our observation in this study. Although three MSCs showed different efficacies on muscle atrophy, all MSCs play an effective role in improve the muscle state from muscle atrophic condition. Among them, UC-MSCs showed slightly higher efficacy than the others. Based on these results, we selected UC-MSCs, and additionally confirmed the effects of MSCs on muscle regeneration and muscle atrophy signaling pathway.

Desmin is an intermediate filament protein expressed during muscle differentiation. It has been employed as a marker of regeneration in muscle diseases, due to expression in regenerating fibers [24]. Herein, a marker of human nuclei was also used to identify the surviving UCMSCs engrafted in the soleus muscle (Figure 6A). The expression of desmin, with its characteristic reticular pattern, was observed in the control group, whereas the pattern was found to be collapsed in the muscle fibers that were damaged due to muscle atrophy caused by HS. However, strong desmin expression was also observed in the UC-MSCs-injected groups (HS+UC and $\mathrm{HR}+\mathrm{UC}$ ). It is therefore safe to assume that muscle regeneration was enhanced in the UC-MSCsinjected groups.

Atrophy of the soleus muscle during HS is accompanied by a decrease in the activation of the anabolic part of the PI3K-AKT signaling pathway and increase in the activation of the catabolic targets FoxO, MAFbx (Atrogin-1) and MuRF-1. The PI3K-AKT pathway is known to play a critical role in activating both the synthesis and degradation of muscle proteins [25]. Although it is mostly thought to promote muscle growth, deactivation of the AKT pathway could also be involved in the regulation of muscle atrophy $[26,27]$. One downstream target of the PI3K-AKT pathway is the forkhead box O (FoxO) family of transcription factors, which is a subgroup of the forkhead family of transcription factors. FoxO is an important regulator of proteolysis, as decreased activation of AKT leads to the FoxO-mediated expression of E3 ubiquitin ligases. The ligases Atrogin-1 (also known as muscle atrophy F-box) and muscle-specific RING finger protein-1 (MuRF-1) are known to be powerful contributors of muscle proteolysis $[28,29]$. From the results, we found that HS-induced muscle atrophy occurs via the PI3K-AKT signaling pathway.

\section{Conclusions}

In conclusion, we anticipate further development of MSCs as a therapy for muscle atrophy. In the short run, MSCs can be used to treat muscle atrophy, while in the long run; they may be applied as treatment for patients suffering from musculoskeletal disorders. To achieve these goals, further studies should follow to elucidate the mechanism of action of MSCs in muscle atrophy. The present study suggests a basis for a new class of medicines for the fast rehabilitation and regeneration of atrophied muscles using MSCs.

\section{Acknowledgement}

This work was supported by the Space Core Technology Development Program, funded by the Ministry of Education Science and Technology (MEST), Korea (Project No.2011-0030754), for which the authors are grateful. 
Citation: Kim MJ, Kim YM, Kim ZH, Heo SH, Kim SM, et al. (2015) Mesenchymal Stem Cells Suppress Muscle Atrophy Induced by Hindlimb Suspension. J Stem Cell Res Ther 5: 266. doi:10.4172/2157-7633.1000266

\section{Conflict of Interests}

The authors declare no competing financial interests.

\section{References}

1. Senf SM, Dodd SL, McClung JM, Judge AR (2008) Hsp70 overexpression inhibits NF-kB and Foxo3a transcriptional activities and prevents skeletal muscle atrophy. FASEB J 22: 3836-3845. [PubMed]

2. Fitts RH, Riley DR, Widrick JJ (2000) Physiology of a microgravity environment invited review: microgravity and skeletal muscle. J Appl Physiol 89: 823-839. [PubMed]

3. Asakura A (2012) Skeletal Muscle-derived Hematopoietic Stem Cells: Muscular Dystrophy Therapy by Bone Marrow Transplantation. J Stem Cell Res Ther S11: 005. [PubMed]

4. Caplan $\mathrm{Al}$ (2007) Adult mesenchymal stem cells for tissue engineering versus regenerative medicine. J Cell Physiol 213: 341-347. [PubMed]

5. Zhang N, Li J, Luo R, Jiang J, Wang JA (2008) Bone marrow mesenchymal stem cells induce angiogenesis and attenuate the remodeling of diabetic cardiomyopathy. Exp Clin Endocrinol Diabetes 116: 104-111. [PubMed]

6. da Silva Meirelles L, Chagastelles PC, Nardi NB (2006) Mesenchymal stem cells reside in virtually all post-natal organs and tissues. J Cell Sci 119: 22042213. [PubMed]

7. Deans TL, Elisseeff JH (2009) Stem cells in musculoskeletal engineered tissue. Curr opin in biotechnol 20: 537-544. [PubMed]

8. Lee JY, Qu-Petersen Z, Cao B, Kimura S, Jankowski R, et al. (2000) Clona isolation of muscle-derived cells capable of enhancing muscle regeneration and bone healing. J cell biol 150: 1085-1100. [PubMed]

9. Dezawa M, Ishikawa H, Itokazu Y, Yoshihara T, Hoshino M, et al. (2005) Bone marrow stromal cells generate muscle cells and repair muscle degeneration. Science 309: 314-317. [PubMed]

10. Morey-Holton ER, Globus RK (2002) Hindlimb unloading rodent model: technical aspects. J Appl Physiol 92: 1367-1377. [PubMed]

11. Perrien DS, Akel NS, Dupont-Versteegden EE, Skinner RA, Siegel ER, et al (2007) Aging alters the skeletal response to disuse in the rat. Am J Physiol Regul Integr Comp Physiol 292: R988-996. [PubMed]

12. Kim SM, Moon SH, Lee Y, Kim GJ, Chung HM, et al. (2013) Alternative xenofree biomaterials derived from human umbilical cord for the self-renewal exvivo expansion of mesenchymal stem cells. Stem Cells Dev 22: 3025-3038. [PubMed]

13. Kocaefe C, Balci D, Hayta BB, Can A (2010) Reprogramming of human umbilical cord stromal mesenchymal stem cells for myogenic differentiation and muscle repair. Stem Cell Rev 6: 512-522. [PubMed]

14. Conconi MT, Burra P, Di Liddo R, Calore C, Turetta M, et al. (2006) CD105 $(+)$ cells from Wharton's jelly show in vitro and in vivo myogenic differentiative potential. Int J Mol Med 18: 1089-1096. [PubMed]

15. Desaphy JF, Pierno S, Liantonio A, Giannuzzi V, Digennaro C, et al. (2010) Antioxidant treatment of hindlimb-unloaded mouse counteracts fiber type transition but not atrophy of disused muscles. Pharmacol Res 61: 553-563. [PubMed]

16. Distefano G, Ferrari RJ, Weiss C, Deasy BM, Boninger ML, et al. (2013) Neuromuscular electrical stimulation as a method to maximize the beneficial effects of muscle stem cells transplanted into dystrophic skeletal muscle. PLoS One 8: e54922. [PubMed]

17. Metter EJ, Talbot LA, Schrager M, Conwit R (2002) Skeletal muscle strength as a predictor of all-cause mortality in healthy men. J Gerontol A Biol Sci Med Sci 57: B359-365. [PubMed]
18. Vieira NM, Zucconi E, Bueno CR Jr., Secco M, Suzuki MF, et al. (2010) Human multipotent mesenchymal stromal cells from distinct sources show different in vivo potential to differentiate into muscle cells when injected in dystrophic mice. Stem Cell Rev 6: 560-566. [PubMed]

19. Krawiec BJ, Frost RA, Vary TC, Jefferson LS, Lang CH (2005) Hindlimb casting decreases muscle mass in part by proteasome-dependent proteolysis but independent of protein synthesis. Am J Physiol Endocrinol Metab 289: E969980. [PubMed]

20. Mujika I, Padilla S (2001) Muscular characteristics of detraining in humans. Med Sci Sports Exerc 33: 1297-1303. [PubMed]

21. Brooks NE, Myburgh KH (2014) Skeletal muscle wasting with disuse atrophy is multi-dimensional: the response and interaction of myonuclei, satellite cells and signaling pathways. Front Physiol 5: 99. [PubMed]

22. Vermaelen M, Sirvent P, Raynaud F, Astier C, Mercier J, et al. (2007) Differentia localization of autolyzed calpains 1 and 2 in slow and fast skeletal muscles in the early phase of atrophy. Am J Physiol Cell Physiol 292: C1723-1731. [PubMed]

23. Masuda S, Hayashi T, Egawa T, Taguchi S (2009) Evidence for differentia regulation of lactate metabolic properties in aged and unloaded rat skeletal muscle. Exp Gerontol 44: 280-288. [PubMed]

24. Hawke TJ, Meeson AP, Jiang N, Graham S, Hutcheson K, et al. (2003) p21 is essential for normal myogenic progenitor cell function in regenerating skeletal muscle. Am J Physiol Cell Physiol 285: C1019-1027. [PubMed]

25. Dupont E, Cieniewski-Bernard C, Bastide B, Stevens L (2011) Electrostimulation during hindlimb unloading modulates PI3K-AKT downstream targets without preventing soleus atrophy and restores slow phenotype through ERK. Am J Physiol Regul Integr Comp Physiol 300: R408-417. [PubMed]

26. Glass DJ (2003) Molecular mechanisms modulating muscle mass. Trends Mo Med 9: 344-350. [PubMed]

27. Kandarian SC, Jackman RW (2006) Intracellular signaling during skeletal muscle atrophy. Muscle Nerve 33: 155-165. [PubMed]

28. Sandri M, Sandri C, Gilbert A, Skurk C, Calabria E, et al. (2004) Foxo transcription factors induce the atrophy-related ubiquitin ligase atrogin-1 and cause skeletal muscle atrophy. Cell 117: 399-412. [PubMed]

29. Gwag T, Lee K, Ju H, Shin $\mathrm{H}$, Lee JW, et al. (2009) Stress and signaling responses of rat skeletal muscle to brief endurance exercise during hindlimb unloading: a catch-up process for atrophied muscle. Cell Physiol Biochem 24 537-546. [PubMed] 